www.medecine-nutrition.org

Éditeur en chef : Alain Baudevin Tarifs d'abonnement (4 num./an) France : $68,60 €$ TTC CEE : $71,65 €$ TTC Hors CEE : 76,22 € TTC Prix du numéro : $20 €$ TTC Pour plus d'informations, contactez le service abonnements : abonnements@edpsciences.org tél. : 0169187575

\section{Publicité}

J. Texier, EDP Sciences jerome.texier@edpsciences.org tél. : 0143293101

\section{EDP Sciences}

17 avenue du Hoggar PA de Courtabœuf, BP 112 91944 Les Ulis Cedex A, France

Directeur de publication : J.-M. Quilbé Production : J. Hamon

ISSN : 0398-7604

e-ISSN : en cours

CPPAP : 0912 T 86980

Imprimé par : LA SIMARRE 37304 Jové-Lès-Tours Dépôt légal : janvier 2011

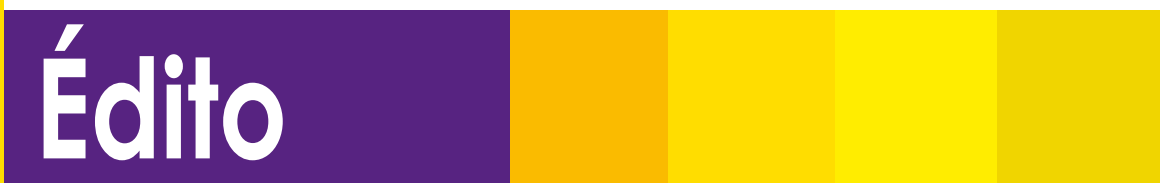

\title{
BONNE ANNÉE!
}

24 rue Joseph Cugnot, BP 10407

Chers lecteurs,

2011 prend le relai de 2010 et nous donne l'occasion de vous souhaiter une excellente année.

Espérons ensemble que cette nouvelle année sera, pour notre domaine de la Nutrition, riche en recherches scientifiques, en événements et nous permettra de vous tenir informé par la publication d'articles complets, documentés, de très bon niveau, tout en restant accessibles.

Traditionnellement, le changement d'année est également l'occasion de remercier tous les auteurs qui ont contribué à la vie du journal. Cette année nous tenons particulièrement à les remercier pour nous avoir renouvelé leur confiance malgré les changements que vous connaissez.

Nous profitons de ces remerciements pour inciter les auteurs désireux de publier, de nous soumettre leur manuscrit à l'adresse mail editorial@edpsciences.org, leur article sera étudié avec le plus grand sérieux.

Dans ce numéro, nous vous proposons plusieurs articles forts intéressants. Ils traitent aussi bien de sujets médicaux tels que " Infection à Helicobacter pylori et cancer gastrique ", "Obésité humaine : vers les approches génomiques fonctionnelles », que de sujets plus sociétaux : "Programme National Nutrition Santé : Bilan et Mesures ", "Plan d'action : Allaitement maternel $1 . .$. Ces articles sont complétés par quelques pages d'actualités.

Nous vous souhaitons bonne lecture et de nouveau une très Bonne Année 2011.

Alain Baudevin

EDP Sciences

(c) EDP Sciences

Tous droits de traduction, d'adaptation et de reproduction pour tous procédés réservés pour tous pays. II est interdit de reproduire, même partiellement, la présente publication sans autorisation de l'éditeur ou du Centre français d'exploitation du droit de copies (loi du jer juillet 1992). 